\title{
Mesenchymal Hamartoma of Liver in a Neonate
}

\author{
Amir-Mohammad Armanian ${ }^{1^{*}}$, Masoud Nazem ${ }^{2}$, Nima Salehimehr ${ }^{3}$ and Babak Nekooie ${ }^{4}$ \\ Disease, Isfahan University of Medical Sciences, Isfahan, Iran \\ ${ }^{2}$ Department of Surgery, School of Medicine, Isfahan University of Medical Sciences, Isfahan, Iran \\ ${ }^{3}$ Department of Health, Almahdi-Mehr Isfahan Higher Education Institution, Isfahan, Iran \\ ${ }^{4}$ Isfahan University of Medical Sciences, Isfahan, Iran
}

${ }^{1}$ Division of Neonatology, Department of Pediatrics, Child Growth and Development Research Center, Research Institute for Primary Prevention of Non-Communicable

*Corresponding author: Amir-Mohammad Armanian, Division of Neonatology, Department of Pediatrics, Child Growth and Development Research Center, Research Institute for Primary Prevention of Non-Communicable Disease, Isfahan University of Medical Sciences, Isfahan, Iran, Tel: 983112355059; Fax: 983112355059; E-mail: armanian@med.mui.ac.ir

Received date: April 19, 2016; Accepted date: May 25, 2016; Published date: May 29, 2016

Copyright: ( $) 2016$ Armanian AM, et al. This is an open-access article distributed under the terms of the Creative Commons Attribution License, which permits unrestricted use, distribution, and reproduction in any medium, provided the original author and source are credited.

\begin{abstract}
Introduction: Mesenchymal hamartoma of liver (MHL) is the second most common benign liver tumor in children. This tumor typically presents as a large benign cystic mass, solid or mix in children less than three years. Prenatally, it may grow quickly to an abnormal size that may lead to death or hydrops. Prenatal suspicion arises by increasing maternal serum $\alpha-F P$ or $H C G$ and polyhydroamnious and can usually identify the case in the last trimester of pregnancy by ultrasound.
\end{abstract}

Case presentation: The patient was a sick 19 day old baby boy. The patient was admitted to the hospital due to the poor feeding, restlessness and nausea. On physical examination, a mass was found on the left side of the abdomen. In the MRI of the abdomen, the heterogeneous multi-lobulated mass was reported in the upper quadrant of the abdomen. Incision and drainage was done and purulent material was drained from a large abscess located in the left lobe of the liver. Finally, the pathology report showed that the infected mesengymal hamartoma of the liver.

Conclusion: In neonatal period MHL can lead to emergency surgery due to fluid or puss accumulation in the cysts. Complete surgical removal is the preferred treatment and histopathological diagnosis helps.

Keywords: Neonate; Abdominal mass; Mesenchymal hamartoma of liver

\section{Introduction}

Mesenchymal hamartoma of liver (MHL) is the second most common benign liver tumor in children [1]. This tumor typically presents as a large benign cystic mass, solid or mix in children less than three years and can be treated with complete removal [2]. Prenatally, it may grow quickly to an abnormal size that may lead to death or hydrops [3]. Clinical presentations of MHL differ from an asymptomatic mass (which accidentally to be discovered during the imaging) to cause symptoms such as abdominal distention, pain, respiratory distress, heart failure, anorexia, vomiting, weight loss, vascular steal, thrombocytopenia, intralesional hemorrhage, pulmonary hypertension, ascites (due to rupture of the tumor), edema of the legs, obstructive jaundice and smooth, large, not painful hepatomegaly $[4,5]$. Prenatal suspicion arises by increasing maternal serum $\alpha \mathrm{FP}$ or HCG and polyhydroamnious and can usually identify the case in the last trimester of pregnancy by ultrasound [4,5]. Associated anomalies may see such as malrotation, BeckwithWiedemann syndrome, biliary atresia and congenital heart disease [4].

\section{Case Presentation}

The patient was a sick 19 day old baby boy, NVD and term. He was the third child of the family with the birth weight of $3400 \mathrm{~g}$. The parents were not related and did not report a specific disease. The patient was admitted to the hospital due to the poor feeding. Three days before the admission of the patient, he was faced with restlessness, poor feeding and nausea without the history of vomiting and fever. The patient was hospitalized suspected with sepsis and were treated with antibiotics, ampicillin and cefotaxime. On physical examination, a mass was found on the left side of the abdomen. Except the abdominal mass, there was no sign of fever, respiratory distress or vomiting. On the admission day, his weight was $3160 \mathrm{~g}$. The ultrasound showed a region of mass like, heterogeneous, mixed echo and multi-lobulated with the dimension of $40 \times 30 \mathrm{~mm}$ in the center of the abdomen, which was the cause of displacement of the spleen and liver. Liver size was reported, span $=55 \mathrm{~mm}$ with normal parenchymal echo and the spleen with the span=75 $\mathrm{mm}$, shaped and normal echo and also normal kidneys. For more review, MRI was recommended.

The test results were as follows:

$\mathrm{WBC}=27300(\mathrm{PMN}=55 \%), \rightarrow 21300, \mathrm{Hb}=10.3 \rightarrow 8.8, \mathrm{HCT}=30.2 \rightarrow$ 27.2, $\mathrm{PLT}=30000 \rightarrow 60000, \mathrm{BUN}=27, \mathrm{Cr}=0.6, \mathrm{Na}=136, \mathrm{k}=4 \mathrm{Ca}=9.5$, $\mathrm{CRP}=7.3, \mathrm{PT}=13, \mathrm{PTT}=29, \mathrm{INR}=1$.

LP was performed for the patient. CSF/C of the patient were negative.

In the course of hospitalization, the patient received blood and platelets due to anemia and thrombocytopenia. On the tenth day of hospitalization, due to the audible heart murmur, echocardiography was performed. There was a small ASD. Sonography of the brain was 
performed, which was normal. In the MRI of the abdomen, the heterogeneous multi-lobulated mass was reported in the upper quadrant of the abdomen with the dimension of $50 \times 50 \mathrm{~mm}$. It was derived from the spleen in the interest of hemangioma (with less probability, it was arising from the left kidney). Due to the deterioration of the patient overall health, the use of vancomycin was started too. Considering the neonatal abdominal mass and causing the lavage and milk intolerance, pediatric surgical consultation was requested and according to the pressure of the mass to the digestive system, barium enema was done for the baby as well. During the infant's hospitalization, occasionally the high blood pressure was also reported. In the performed barium enema, there was no pathological point. Finally, incision and drainage was done and purulent material was drained from a large abscess located in the left lobe of the liver, with the adhesion to the spleen and colon. By laparotomy, the left lobe of the liver and transverse colon were liberated. Left hepatic lobectomy was performed. Finally, the pathology report showed that the infected mesengymal hamartoma of the liver. After completing the course of antibiotics patient was discharged with a good general condition and full breastfeeding.

\section{Discussion}

The incidence of liver tumors in the general population is about five percent of congenital tumors that can be caused by mesenchymal or endodermal tissues. This provides a wide range of malignant and benign tumors [6]. Hepatic hemangioma, MHL and hepatoblastoma are the most common primary tumors in the neonatal period [5]. MHL more commonly involve the right lobe of the liver (75\%). It is usually multi-lobulated and in twenty percent of the cases is pedanculated (leggy) [4]. 20\% of the cases occur in neonates and may have a rapid growth after the birth, which is due to fluid accumulation $[4,5]$. The rapid accumulation of fluid in the cyst could result the pressure on the lungs and causing respiratory distress. Tumors may be very large and sometimes up to 20 to $30 \mathrm{~cm}$ in diameter and the need for a complete tumor removal surgery [4]. Incomplete spontaneous regression or malignant transformation may occur in it [4]. Malrotation can occur due to the rotation of the large cyst [4]. MHL may be due to ductal plate malformation, vascular disorders or neoplastic damage [4,5]. However, calcification, severe necrosis and intra-lesional hemorrhage are rare. MHL is typically a multi-locular round shape without capsule, which is not communicated with biliary tract and is surrounded by an irregular margin of the liver parenchyma, bile duct and blood vessels [4]. Simple abdominal radiographs may show the calcification and a large soft tissue mass with bowel dislocation. Ultrasound, CT scan and MRI of MHL demonstrate a multilocular cystic tumor with varying amounts of solid tissue [4]. By using the ultrasound, mobile thin walls or hyper-echo nodules in the cyst are strongly in favor of the diagnosis of MHL. Differential diagnosis of abdominal cystic mass in infants are included mesenteric Cyst, duplication cyst, hepatoblastoma, teratoma, linfangioma, colloid cyst, neoplastic solitary cyst and renal Cyst
[4,7-11]. Mesenteric and duplication cysts are mobile but the duplication has specified internal walls [9]. Colloid cyst, according to the location of the lesion in relation to the liver is denoted by proximal dilatation of the bile duct and jaundice. Non-parasitic solitary hepatic cysts which are rare in neonates could be congenital or acquired. These are more common in females and involvement cubic epithelium was observed. $[9,10]$. Diagnosis of sub-hepatic cystic large pedicle (variant of MHL) before the surgery of can be difficult [4,7-13]. Infection and relapse are common and occur followed by percutaneous drainage of the large cyst. Complete removal of MHL by the laparoscopic surgery is the treatment of choice in neonates $[4,5]$.

\section{Conclusion}

In neonatal period MHL can lead to emergency surgery due to fluid or puss accumulation in the cysts. Complete surgical removal is the preferred treatment and histopathological diagnosis helps.

\section{References}

1. Otero AO, de Prada Vicente I, Herrera JG, Cuadrilleros DA (2012) Hepatic mesenchymal hamartoma in a neonate with a Bochdalek diaphragmatic hernia: A case report. Journal of Pediatric Surgery 47: 45-48.

2. Ramareddy RS, Alladi A (2012) Neonatal mesenchymal hamartoma of liver: An unusual presentation. J Clin Neonatol 1: 211-213.

3. Kodandapani S, Pai MV, Kumar V, Pai KV (2011) Prenatal Diagnosis of Congenital mesenchymal hamartoma of liver: A Case Report. Case Reports in Obstetrics and Gynecology.

4. Stringer MD, Alizai NK (2005) Mesenchymal hamartoma of the liver: A systematic review. J Pediatr Surg 40: 1681-1690.

5. Isaacs H Jr (2007) Fetal and neonatal hepatic tumors. J Pediatr Surg 42: 1797-1803.

6. Cignini P, Coco C, Giorlandino M, Bagolan P, Morini F, et al. (2007) Fetal hepatic mesenchymal hamartoma: A case report. J Prenat Med 1: 45-46.

7. Wootton-Gorges SL, Thomas KB, Harned RK, Wu SR, Stein-Wexler R, et al. (2005) Giant cystic abdominal masses in children. Pediatr Radiol 35: 1277-1288.

8. Avni EF, Rypens F, Donner C, Cuvelliez P, Rodesch F (1994) Hepatic cysts and hyperechogenicities: Perinatal assessment and unifying theory on their origin. Pediatr Radiol 24: 569-572.

9. Quillin SP, McAlister WH (1992) Congenital solitary nonparasitic cyst of the liver in a newborn. Pediatr Radiol 22: 543-544.

10. Shankar SR, Parelkar SV, Das SA, Mathure AB (2000) An antenatallydiagnosed solitary, non-parasitic hepatic cyst with duodenal obstruction. Pediatr Surg Int16: 214-215.

11. Berg C, Baschat AA, Geipel A, Krapp M, Germer U, et al. (2002) Firsttrimester diagnosis of fetal hepatic cyst. Ultrasound Obstet Gynecol 19: 287-289.

12. von Schweinitz D (2003) Neonatal liver tumours. Semin Neonatol 8: 403-410.

13. Okumura M, Liao AW, Brizot Mde L, Zugaib M, da Silva MM (2008) Sonographic detection of a giant solitary hepatic cyst in utero. J Ultrasound Med 27: 1663-1665. 